\title{
Psychometric Properties of the Albanian Version of the Adult Self-Report for Ages 18-59
}

\section{Elvisa Sokoli}

University of Tirana, Tirana, Albania; Email: elvis.sokoli@gmail.com

\section{Blerta Bodinaku}

University of Tirana, Tirana, Albania; Email: bbodinaku@gmail.com

Joana Paço

University of Tirana, Tirana, Albania; Email: joanapaco1@gmail.com

Ornela Gjergji

University of Tirana, Tirana, Albania; Email: ornela_gjergji@hotmail.com

\section{Ledja Çala}

University of Tirana, Tirana, Albania; Email: ledjacala13@gmail.com

\section{Doi:10.5901/ajis.2016.v5n1p51}

\section{Abstract}

\begin{abstract}
The Achenbach System of Empirically Based Assessment (ASEBA) constitutes an all-inclusive evidence-based assessment system assessing competencies, adaptive functioning, social, emotional, and behavior problems of individuals from age of 1 1/2-90 years. ASEBA instruments are consistently used for clinical assessment, outcome evaluations, epidemiological survey and research. Its approach is also widely used for multicultural research. ASEBA instruments are translated and used in different cultures over the world in different settings such as mental health, medical, child and family, school, public health agencies etc. (Achenbach \& Rescorla, 2015). Adult Self-Report for Ages 18-59 (ASR), an ASEBA self-report measure, are one of the most widely used and well-known psychometric measures for assessing adults' adaptive functioning and emotional and behavior problems (Achenbach \& Rescorla, 2003). In order to culturally adapt, standardize and validate the instrument for the Albanian context the reliability and validity of the ASR was examined using a national representative sample of 750 Albanian adults. In order to reach reliability, internal consistency analyses were performed. Empirically based syndrome scales of ASR in the Albanian sample ranged from .50 to .96 from the Cronbach's alphas obtained with a mean alpha of .80, falling within the "good to excellent" range except for the Thought Problems syndrome scale having an alpha of .50. Confirmatory factor analysis (CFA) indicated a good model fit of ASR scales in the Albanian sample. Furthermore, Albanian norm scores and cut-off scores have been generated for the Albanian version of ASR. The article provides evidence regarding the psychometric properties and utility of ASR in the Albanian adult population for clinical assessment, outcome evaluations and research purposes.
\end{abstract}

Keywords: ASEBA, ASR, empirically based syndrome scales; DSM-oriented scales, psychological functioning.

\section{Introduction}

Measuring adults' psychological functioning is usually a difficult task. Consensus on which assessment tools should be used is yet to be reached, however most researches argue that the assessment of adults' psychological functioning relies mostly on the reports of their own functioning via self-reports, questionnaires and interviews, (Achenbach, Berstein \& Dumenci, 2005). There is a multitude of evidence demonstrating that self-report inventories are among the most standardizable tools of data collection which exclude the effect of interviewers and minimize the social desirability. Also self-reports have many advantages such as: easily distributed, low cost, more time efficient and ability to reflect the respondent's own functioning (Schuman \& Presser, 1996).

For these reasons they are of essential importance for use in mental health services, schools, medical settings, public health agencies, multicultural assessments, epidemiological surveys and research (Kessler, et.al, 2000). However, 
the necessity to empirically evidence their properties in order to use them in such settings is of outmost importance and needs to be emphasized. Moreover, due to their sensitivity to culture and language, they must be standardized and validated in order to reflect the characteristics of the target group on specific populations (Meyer, et.al, 2001).

In adult mental health services worldwide, the Achenbach System of Empirically Based Assessment (ASEBA) is one of the most widely used and well-known psychometric measures for assessing adult psychological functioning (Henriksen \& Bilenberg, 2012), including adaptive functioning and emotional, social and behavior problems (Achenbach, 2006). ASEBA offers mental health professionals practical measures for the assessment of adult psychopathology that appear to be meaningfully used across multi-societies due to their cultural generalizability (Ivanova et al., 2014).

An investigation of the recent state of mental health settings in Albania indicates the lack of reliable and valid psychometric assessment investigating adults' psychological functioning until recently (Bodinaku, 2014). The clients' assessment relied only on the clinical judgment of mental health practitioners. Moreover the few assessment tools that psychosocial professionals used were subjective and contain no empirically based standards of measurement which do not guarantee standardized and valid assessment procedures (WHO, 2002; OSFA, 2012). There has been a neglect to research and validate instruments in the mental health field to appropriately diagnose, interview, and treat adults. Because of the increased diversity of population due to the mobility in Albania, as in many countries, healthcare researchers must have access to reliable, standardized and cross-validated measures, in order to understand more deeply the total functioning of individuals. Systematic application of validated measurements by the mental healthcare system in developing countries and their association with the adult's psychological functioning across the cultures becomes the key to find significant targets for intervention and prevention in adults mental health (Sousa \& Rojanasrirat, 2011). Due to the necessity of improving the mental health practice in Albania, Bodinaku (2014) standardized and validated two psychometric instruments: CORE-OM and SCL-90-R, which are currently used by the psychosocial professionals. Nevertheless, there is a clear need to establish the use of more cross-culturally validated measures in Albania.

Aiming to meet the need for a standardized, validated psychometric measure which can be used in mental health services and research the goal of this study is to culturally adapt and investigate the psychometric properties of Adult Self-Report for ages 18-59 in the Albanian population.

\section{Methods}

\subsection{Participants}

Normative data were collected in an epidemiological cross-sectional design. A total of $\mathrm{N}=750$ participants aged 18-59 years (mean age $M=37.32, S D=12.75$ ) from nationally demographically representative distribution participated in this study. The sample represents the population of Albania regarding three demographic variables: gender, age and Albanian geographical units based on Census 2011 data (INSTAT, 2011). The adults' participating in this study were stratified by two age groups (18-35 and 36-59 years old), gender, Albanian counties (12) and Albanian zones (urban and rural) in order to guarantee the representativeness of the Albanian population. The participants sample comprised $n=377$ men $(50.3 \%)$ and $n=373$ women $(49.7 \%)$. They were all invited to self- report on their psychological functioning by completing ASR.

\subsection{Instrument}

The Adult Self-Report for Ages 18-59 (ASR) for adults was introduced in Albania in 2015 and will be currently used in mental health services, such as clinics, community mental health centers, substance abuse treatment settings, educational settings, children's services, forensic settings such as courts, division programs, medical settings and outcome evaluations (Achenbach \& Rescorla, 2015). ASR has proven to be a reliable and valid measure for gathering self-reported information regarding adaptive and maladaptive psychological functioning of adults (van der Ende, Verhulst, \& Tiemeier, 2012; Saavedra, Silverman, Morgan-Lopez, \& Kurtines, 2010; Buysse et al., 2010). The ASR is a self-report questionnaire for ages 18-59 that assesses behavioral, emotional, and social problems, including also adaptive functioning, substances use and personal strengths (Achenbach \& Rescorla, 2003).

The questionnaire has to be completed by the adult that is being assessed. The ASR is divided into two main sections and the norms for all the scales are constructed for two age groups: 18-35 years and 36-59 years (Achenbach \& Rescorla, 2003). The first part of the instrument assesses the adult's level of adaptive functioning. The second part of it 
consists of 123 problem items for which the adult rates his/her emotional, social and behavioral problems concerning each item on a 3-point Likert scale with "0" corresponding to "not true," "1" corresponding to "somewhat or sometimes true," and "2" corresponding to "very true or often true." Via factor-analytic methods, eight empirically based syndrome scales are derived: Anxious/Depressed (18 items), Withdrawn (9 items), Somatic Complaints (12 items), Thought Problems (10 items), Attention Problems (15 items), Aggressive Behavior (15 items), Rule-Breaking Behavior (14 items), and Intrusive (6 items) (Achenbach \& Rescorla, 2003). Furthermore, the syndromes are grouped into two main grouping of problems: (1) "Externalizing Problems" including: aggressive behavior, rule-breaking behavior, and intrusive and (2) "Internalizing Problems" including anxious/depressed, withdrawn, somatic complaints. Summation of all the syndrome scales yields a "Total Problems" score. The questionnaire also includes a Critical Items scale (19 items), an Other Problem scale (21 items), a Substance Use scale (3 items) and a Social Desirability scale (11 items) (Achenbach \& Rescorla, 2003). Moreover, diagnostically meaningful scales, so-called Diagnostic and Statistical Manual of Mental Disorders (DSM) oriented scales have been developed to increase the clinical advantage of the ASEBA instruments for adults. Some of the ASR items form 6 DSM-oriented scales. These scales contain problem items that are frequent among patients with corresponding DSM diagnoses such as: depressive problems (14 items), anxiety problems (7 items), somatic problems (9 items), avoidant personality problems (7 items), attention deficit/hyperactivity problems (13 items) and antisocial personality problems (20 items) (Achenbach \& Rescorla, 2003).

\subsection{Translation and back-translation procedures}

In order for an instrument to be used in another language, it must be forwardly translated, back translated, culturally adapted and validated in the culture of interest (Wild et al., 2005). These procedures are consistent with the Guidelines for Translating and Adapting Tests (International Test Commission [ITC], 2010), and the Standards for Educational and Psychological Testing (AERA, APA, NCME, 1999). The forward translation of ASR was performed by an interdisciplinary group composed of psychologists, public health professionals, education specialists, English professors, graduate students in psychology and a person having no background in any of the above mentioned fields. The translation focused on retaining the original wording and containing the meaning of the Albanian language. The group of professionals appraised the ASR item-by-item and provided detailed feedback on each item's wording and meanings. This feedback was then included in the ASR questionnaire in order to produce the Albanian version. Even though the Albanian language contains dialects that vary somewhat by geographic regions, the translation was based on the standardized Albanian language (1972) which can be generalizable to all Albanian-speaking regions. A native English speaker translator who knew Albanian very well then performed a back-translation of the Albanian version of ASR. The original English and the back translation were reviewed by the experts and researchers to revise ambiguous or misleading items. Finally, the group of experts revised item by item all forward translations and back-translation, leading to the final Albanian version of ASR. This procedure provided a culturally sensitive version of a widely used instrument to measure adult's psychological functioning. The standardized Albanian version of the instrument remained as close to the original English version as possible which ensured validity and reliability when comparing English-speaking populations with Albanian-speaking populations (Ivanova, et al., 2014).

\section{Results}

The data in this study were analyzed through the Statistical Package for Social Sciences (SPSS), version 21.0. A total of $\mathrm{N}=750$ participants aged 18-59 years completed ASR. Response rates were excellent for ASR with no excluded forms from the analysis.

In order to check the reliability of the Albanian version of the ASR, we computed the internal consistency, using the Cronbach Alpha coefficient as an indicator. For the Albanian version of ASR, alpha coefficient for Total Problems was .96 , and alphas for Internalizing and Externalizing Problems were $>.91$ and $>.93$, showing an excellent internal consistency (Cronbach, 1951; 1971). The mean alpha scores for the other scales ranged from .50 to .89. Particularly, Aggressive Behavior, Anxiety/Depression, Attention Problems, Rule-Breaking Behavior and Somatic Complaints had very good internal consistency (alphas ranged between .80 and .89). Whereas Withdrawn, Intrusive, Social Desirability and Critical Items had acceptable internal consistency (alpha values between .72 and .79). Other Problems (alpha .68) showed questionable internal consistency while Thought Problems had the lowest internal consistency (alpha .50).

Among the DSM-oriented scales, Depressive Problems, Attention Deficit/ Hyperactivity problems and Antisocial Personality Problems had very good internal consistency (alpha values between .81 and .86). Somatic Complaints and 
Inattention, Hyperactivity-Impulsivity subscales had acceptable internal consistency (alphas values between .70 and .72). Whereas Anxiety Problems and Avoidant Personality Problems had the lowest internal consistency (alphas values between .62 and .65). With regard to reliability, our analysis confirmed internal consistency, the Cronbach's a for the ASR scales in the sample ranged from .50 to .96 with a mean a of .80, falling within the "good" range (Cronbach, 1951; 1971).

As for the validity, which refers to the accuracy with which instruments assessed what they were supposed to assess (Achenbach and Rescorla, 2003), we used as method for establishing construct validity through testing the convergent validity. Convergent validity refers to the degree to which the scores of an instrument are consistent with hypotheses based on the assumption that the instrument validly tests whether constructs that should be related, are related (Cronbach \& Meehl, 1955). One way of establishing convergent validity was to check the scale's internal structure by calculating the correlation between the Albanian version of ASR scales (Zasepa \& Wolanczyk, 2011). The results of convergent validity estimations ranged between medium and high Pearson correlations. The empirically based syndrome scales of ASR demonstrate significant correlation with each other and with the grouping of syndromes: Internalizing, Externalizing and Total Problems.

The results showed significant correlations also with DSM-oriented scales. The syndrome scales of Anxious/Depressed, Somatic Complaints and Withdrawn demonstrated higher Pearson correlations with Internalizing Problems (ranging from .77 to .94) than with the Externalizing Problems (ranging from .34 to .56). On the contrary, Aggressive Behavior, Rule-Breaking Behavior and Intrusive correlate more highly with Externalizing Problems (ranging from .76 to .95) than with Internalizing Problems (ranging from .28 to .59). Medium to high correlations were found between all syndromes scales and Total Problems (ranging from .62 to .89).

High Pearson correlations were found between the syndrome scales and the DSM-oriented scales which measure similar types of problems. High significant correlations are shown between the Somatic Complaints scale and the DSMoriented scale Somatic Problems ( $r=.96)$. Also high Pearson correlations were found between Anxious/Depressed syndrome scale and two DSM-oriented scales: Depression Problems ( $r=.87)$ and Anxiety Problems ( $r=.76)$; Withdrawn and Avoidant Personality Problems ( $r=.84)$; Attention Problems and AD/H Problems ( $r=.85)$; Aggressive Behavior, RuleBreaking Behavior, Intrusive and Antisocial Personality Problems (ranging from .59 to .93).

Confirmatory factor analysis (CFA), were used to test the fit of self-ratings in the Albanian sample to the eight syndrome model which derived from 123 items, 99 of which loaded significantly on the syndromes. We hypothesized that the ASR syndrome model would be supported by our CFAs of self-ratings by adults in the Albanian sample. The primary fit (Index Root Mean Square Error of Approximation) showed good fit for the total sample (.026). RMSEA were computed for men and women subsamples. With values ranging from .024 for men and .022 for women, RMSEA indicated a good model fit for both genders in the Albanian sample. Comparative Fit Index (CFI) and Tucker Lewis Index (TLI) were also computed secondary to the RMSEA. We referred to the ranges presented by Marsh, Hau and Wen (2004) to estimate the CFI and TLI values. CFI values ranged from .914 for the total sample; .920 for men and .932 for women. TLI values ranged from .911 for the total sample; .917 for men and .930 for women indicating a good model fit of ASR scales in the Albanian sample and subsamples.

Mean values \pm standard deviations (SD) for Empirically Based scales, Other Problems, Critical Items and Social Desirability Items scales, DSM-Oriented scales, Adaptive Functioning scales and Substance Abuse scales were calculated. Independent-samples t-tests were conducted to compare gender differences for all ASR scales. Results for Empirically Based Syndromes scales, Other Problems, Critical Items and Social Desirability Items scales are presented in Table 1. Significant differences between men and women were found for the Internalizing Problems scale: Anxiety/Depression \& Somatic Complains; Externalizing Problems scale: Rule-Breaking Behaviors \& Intrusive; and Thought Problems scale.

Men self-reported higher scores on Externalizing Problems scale, Rule-Breaking and Intrusive Behavior than women. While women self-reported higher scores on Internalizing Problems scale, Anxiety/Depression, Somatic Complaints and Thought Problems.

Nearly significant differences between men and women were self-reported for the Total Problems scale, Withdrawn and Other Problems scales whereas no significant differences between men and women were self-reported for the Attention Problems, Aggressive Behaviors, Critical Items and Social Desirability Items scales. 
Table 1. ASR score Means and Standard Deviations for men and women in the representative sample $(n=750)$.

\begin{tabular}{|c|c|c|c|c|c|c|c|c|}
\hline & $\begin{array}{l}\text { Men } \\
\mathrm{N}=377\end{array}$ & & $\begin{array}{l}\text { Women } \\
\mathrm{N}=373\end{array}$ & & ES & $\begin{array}{l}\text { t-test } \\
\mathrm{df}=748\end{array}$ & & \\
\hline & m & $\mathbf{s}$ & $\mathbf{s}$ & $\mathbf{s}$ & d & $t$ & $\mathrm{p}$ & \\
\hline ASR- Total Problems & 46.98 & 27.04 & 50.62 & 28.59 & -.13 & -1.789 & .074 & $\sim$ \\
\hline ASR- Externalizing Problems & 12.22 & 11.18 & 10.31 & 9.06 & .19 & 2.570 & .010 & * \\
\hline ASR- Internalizing Problems & 15.50 & 9.59 & 19.76 & 12.02 & -.39 & -5.361 & .000 & *** \\
\hline ASR- Anxiety/Depression & 8.40 & 5.09 & 10.91 & 6.68 & -.42 & -5.803 & .000 & 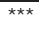 \\
\hline ASR- Withdrawn & 4.44 & 3.01 & 4.88 & 3.26 & -.14 & -1.914 & .056 & $\sim$ \\
\hline ASR- Somatic Complaints & 2.66 & 3.05 & 3.96 & 3.97 & -.37 & -5.044 & .000 & *** \\
\hline ASR- Thought Problems & 1.77 & 1.53 & 2.15 & 2.17 & -.20 & -2.763 & .006 & ** \\
\hline ASR- Attention Problems & 7.03 & 4.98 & 7.38 & 4.95 & -.07 & -.955 & .340 & n.s \\
\hline ASR- Aggressive Behaviors & 5.89 & 5.85 & 5.34 & 5.09 & .10 & 1.388 & .165 & n.s \\
\hline ASR- Rule-Breaking Behaviors & 3.49 & 4.05 & 2.50 & 2.94 & .28 & 3.836 & .000 & 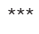 \\
\hline ASR- Intrusive & 2.84 & 2.47 & 2.48 & 2.33 & .15 & 2.075 & .038 & * \\
\hline ASR- Other Problems & 10.46 & 4.53 & 11.02 & 4.81 & -.12 & -1.664 & .096 & $\sim$ \\
\hline ASR- Critical Items & 3.25 & 3.62 & 3.61 & 4.08 & -.09 & -1.295 & .196 & n.s \\
\hline ASR- Social Desirability Items & 16.95 & 3.74 & 17.25 & 3.51 & -.08 & -1.101 & .271 & n.s \\
\hline
\end{tabular}

Results for ASR DSM-Oriented scales are presented in Table 2. No significant differences between men and women were found for the $\mathrm{AD} / \mathrm{H}$ Problems and its subscales (Inattention and Hyperactivity-Impulsivity). Significant differences between men and women were found for all the other DSM-Oriented scales. Women self-reported higher symptoms than men in the Depressive Problems, Anxiety Problems, Somatic Problems and Avoidant Personality Problems scales, while men self-reported higher scores on Antisocial Personality Problems.

Table 2. ASR score Means and Standard Deviations for men and women in the representative sample $(n=750)$.

\begin{tabular}{|c|c|c|c|c|c|c|c|c|}
\hline & $\begin{array}{l}\text { Men } \\
\mathrm{n}=377\end{array}$ & & $\begin{array}{l}\text { Women } \\
n=373\end{array}$ & & ES & $\begin{array}{l}\text { t-test } \\
\mathrm{df}=748\end{array}$ & & \\
\hline & $\mathrm{m}$ & $\mathbf{s}$ & $\mathrm{m}$ & $\mathbf{s}$ & d & $t$ & $p$ & \\
\hline ASR- Total Problems & 26.24 & 16.03 & 28.47 & 16.92 & -.14 & -1.853 & .064 & $\sim$ \\
\hline ASR- Depressive Problems & 4.13 & 3.72 & 5.57 & 4.74 & -.34 & -4.617 & .000 & *** \\
\hline ASR- Anxiety Problems & 5.72 & 2.16 & 7.01 & 2.65 & -.53 & -7.285 & .000 & *** \\
\hline ASR- Somatic Problems & 1.93 & 2.20 & 2.86 & 2.86 & -.36 & -4.972 & .000 & *** \\
\hline ASR- Avoidant Personality Problems & 2.79 & 2.19 & 3.58 & 2.68 & -.32 & -4.434 & .000 & *** \\
\hline ASR- AD/H Problems & 6.46 & 4.67 & 5.97 & 4.39 & .11 & 1.483 & .139 & n.s \\
\hline ASR- - Inattention & 3.35 & 2.61 & 3.14 & 2.56 & .08 & 1.102 & .271 & n.s \\
\hline ASR- - Hyperactivity-Impulsivity & 3.11 & 2.58 & 2.83 & 2.40 & .11 & 1.555 & .120 & n.s \\
\hline ASR- Antisocial Personality Problems & 5.21 & 5.75 & 3.50 & 4.11 & .34 & 4.704 & .000 & *** \\
\hline
\end{tabular}

n.s, no significant ( $p>.1)$, nearly significant $\sim p \leq .1, \quad{ }^{*} p \leq .05, \quad{ }^{*} p \leq .01, \quad{ }^{\star * *} p \leq .001$, two-sided

Results for ASR Adaptive Functioning and Substance Abuse scales are presented in Table 3. Significant differences between men and women were found for all the Substance Abuse scales and the Spouse/Partner scale- an Adaptive Functioning scale. Men self-reported as being more abusive than women with tobacco, alcohol and drugs. Also men selfreported having better relationship with their spouse/partner compared to women. No significant differences were found in the other Adaptive Functioning scales.

Table 3. ASR score Means and Standard Deviations for men and women in the representative sample $(n=750)$.

\begin{tabular}{lcccccccccc}
\hline & \multicolumn{1}{c}{ Men } & \multicolumn{1}{c}{ Women } & ES & t-test & & \\
\cline { 2 - 12 } & $\mathbf{n}$ & $\mathbf{m}$ & $\mathbf{s}$ & $\mathbf{n}$ & $\mathbf{m}$ & $\mathbf{s}$ & $\mathbf{d}$ & $\mathbf{t}$ & $\mathbf{d f}$ & $\mathbf{P}$ \\
\hline ASR Friends scale & 376 & 7.70 & 2.37 & 373 & 7.52 & 2.60 & .07 & 1.016 & 747 & .310 n.s \\
ASR Spouse/Partner scale & 255 & 4.80 & 3.06 & 246 & 4.02 & 3.50 & .24 & 2.630 & 499 & .009 \\
ASR Family scale & 376 & 1.82 & .31 & 370 & 1.83 & .31 & -.04 & -.599 & 744 & .550 n.s \\
ASR Job scale & 256 & .59 & 2.04 & 194 & .86 & 1.73 & -.14 & -1.466 & 448 & .143 n.s \\
\hline
\end{tabular}




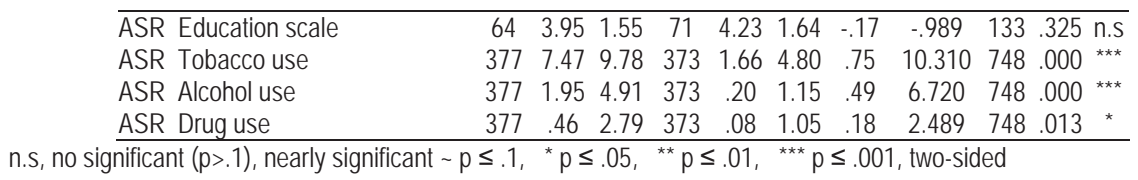

\section{Discussion}

The ASR is one of the most widely used assessment instruments for adult emotional, social and behavioral problems and has a proven multicultural factor structure in many developed societies and a few developing societies (Achenbach \& Rescorla, 2015). The aim of this study was to culturally adapt and investigate the psychometric properties of Adult SelfReport for ages 18-59 in the Albanian population. The lack of standardized psychometric measures has made this study necessary.

One of the most important factors when evaluating the quality of the results from this study refers to the study population's representativeness which was achieved. Our findings are based on data obtained from a population-based sample of nationally representative adults' aged 18-59 years. In this study we determined the psychometric properties of the Albanian version of the Adult Self Report, which in the original version on the ASEBA adult's forms, has shown good psychometric properties (Achenbach \& Rescorla, 2015). To assess the reliability of the measure, the internal consistency was conducted. Most of the scales of the original form for adults had adequate internal consistency. The mean Cronbach alpha is .80 for the ASR syndrome scales, and .84 for the DSM-Oriented ASR scales. The convergent validity has been satisfactorily demonstrated for the Albanian version of ASR. All Pearson correlations between scales are significant, ranging between .62 and .94 in the Albanian version of ASR as in the original version.

This study tested the generalizability of the eight syndrome scales of ASR for assessing adult psychopathology in Albania. In our nationally representative sample the eight syndrome model converged, RMSEA and secondary indices (CFI and TLI) indicated a good model fit. The results supported the eight syndrome model of ASR scales in the Albanian sample and gender subsamples.

We compared our adults' sample results on all ASR scales with respect to their gender. The mean level of selfreported Total Problems by the participants was nearly the same for both genders. There were differences between Internalizing and Externalizing Problems between Albanian men and women. Albanian women self-reported higher Internalizing Problems (including Anxiety/Depression and Somatic Complaints) and Thought Problems. In contrary Albanian men self-reported higher levels of Externalizing Problems (including Rule-Breaking Behavior and Intrusive) and on all the Substance Abuse scales.

This study shows some limitations. Additional other types of reliability and validity analyses need to be performed. Further studies using the Albanian version of ASR should take into consideration the comparison between referred and non-referred Albanian representative samples.

After translation, standardization and validation the ASR for adults of ages 18-59 was introduced in Albania. Clinical constructs of adult psychopathology were supported in the Albanian sample. Norms scores and cut-off scores have been generated separately for men and women of ages 18-35 and 36-59 years. The questionnaire is now available to be used by the Albanian mental health professionals to assess adaptive functioning and emotional, social and behavioral problems of the Albanian adults. The constructs can also be used in further research and training services.

\section{References}

Achenbach, T.M. (2006). As others see us: Clinical and research implications of cross-informant correlations for psychopathology. Current Directions in Psychological Science, 15, 94-98.

Achenbach, T.M., \& Rescorla, L.A. (2003). Manual for the ASEBA adult forms \& profiles. Burlington, VT: University of Vermont, Research Center for Children, Youth, and Families.

Achenbach, T.M., \& Rescorla, L.A. (2015). Multicultural Supplement to the Manual for the ASEBA Adult Forms \& Profiles. Burlington, VT: University of Vermont, Research Center for Children, Youth, \& Families.

Achenbach, T.M., Bernstein, A., Dumenci.L. (2005). "DSM-Oriented Scales and Statistically Based Syndromes for Ages 18 to 59 : Linking Taxonomic Paradigms to Facilitate Multi-taxonomic Approaches". Journal of Personality Assessment, 84(1), 49-63.

American Educational Research Association, American Psychological Association, and National Council on Measurement in Education. (1999). Standards for educational and psychological testing. Washington, DC: American Educational Research Association.

Bodinaku, B. (2014). Translation, validation and standardization of the Albanian version of the SCL-90-R (Symptom Checklist-90Revised) and CORE-OM (Clinical Outcomes in Routine Evaluations - Outcome Measure). Unpublished doctoral dissertation. 
Sigmund Freud Private University, Vienna.

Buysse, C.M.P., Vermunt, L.C.A.C., Raat, H., Hazelzet, J.A., Hop, W.C.J., Utens, E.M.W.J., Joosten, K.F.M. (2010). Surviving meningococcal septic shock in childhood: Long-term overall outcome and the effect on health-related quality of life. Critical Care, 14, R124.

Cronbach, J.L., \& Meehl, E. P. (1955). Construct validity in psychological tests. Psychological Bulletin, 52 (4).

Cronbach, L. J. (1951). Coefficient alpha and the internal structure of tests. Psychometrika, 16(3), 297-334.

Cronbach, L.J. (1971). Test validity. In R. L. Thorndike (Ed.), Educational measurement (2nd ed.pp.430-507). Washington, DC: American Council on Education.

Henriksen.J., P.F., \& Bilenberg.N. (2012). New Danish standardization of the Child Behaviour Checklist. Dan Med J :59 (7): A4462.

International Test Commission (2010). International Test Commission Guidelines for Translating and Adapting Tests. Retrieved from http://www.intestcom.org

Ivanova, Y. M., Achenbach, M. Th., Rescorla, A. L., Turner, V. L., Sokoli. E. et.al., (2014). Syndromes of collateral-reported sychopathology for Ages 18-59 in 18 Societies. International Journal of Clinical and Health Psychology (2015) 15, $18--28$.

Kessler, R. C., Wittchen, H.U., Abelson, J., Zhao, S., Stone, A. A., Turkkan, J. S. (2000). Methodological issues in assessing psychiatric disorders with self-report.In the science of self-report: Implications for research and practice. (pp. 229-255). Mahwah, NJ US: Lawrence Erlbaum Associates Publishers.

Instituti i Gjuhësisë dhe Letërsisë të Universitetit Shtetëror të Tiranës (1972). Kongresi i Drejtshkrimit të Gjuhës Shqipe.

Marsh, H. W., Hau, K. T., \& Wen, Z. (2004). In search of golden rules: comment on hypothesis-testing approaches to setting cutoff values for fit indexes and dangers in overgeneralizing Hu and Bentler's (1999) findings. Structural Equation Modeling, 11, 320341.

Meyer, G. J., Finn, S. E., Eyde, L. D., Kay, G. E., Moreland, L. L., Dies, R. R., Eisman, E. J., Kubiszyn, T. W., \& Reed, G. M. (2001). Psychological Testing and Psychological Assessment. A Review of Evidence and Issues. American Psychologist. 56(2): 128-165.

National Institute of Statistics (2011). Population and housing census 2011. Tiranë:

Open Society Foundation for Albania (2012). Assessment of mental health care needs at national level. Tirana: Author.

Saavedra, L.M., Silverman, W.K., Morgan-Lopez, A.A., Kurtines, W.M. (2010). Cognitive behavioral treatment for childhood anxiety disorders: Long-term effects on anxiety and secondary disorders in young adulthood. Journal of Child Psychology and Psychiatry, 51, 924-934.

Sousa.D.V., \& Rojanasrirat.W. (2011). Translation, adaptation and validation of instruments or scales for use in cross-cultural health care research: a clear and user-friendly guideline. Journal of Evaluation in Clinical Practice, 17. 268-274.

Schuman, H., \& Presser, S. (1996). Questions and answers in attitude surveys: Experiments on question form, wording and context. Newbury Park, CA: Sage.

Van der Ende, J., Verhulst, F.,\& Tiemeier, H. (2012). Agreement of informants on emotional and behavioral problems from childhood to adulthood. Psychological Assessment, 24, 293-300.

Wild, D., Grove, A., Martin, M., Eremenco, S., McElroy, S., Verjee-Lorenz, A., Erikson, P. (2005). Principles of Good Practice for the Translation and Cultural Adaptation Process for Patient-Reported Outcomes (PRO) Measures: Report of the ISPOR Task Force for Translation and Cultural Adaptation. Value Health, 8: 94-104

World Health Organization. (2002). World report on violence and health. Geneva: Author.

Zasepa E., Wolanczyk, T. (2011). Assessment of problem behaviors in adults' population. Evaluation of psychometric characteristics of the Polish adaptation Adult Self-Report (ASR) and Adult Behavior Checklist (ABCL). International Journal of Child Health \& Human Development, 4, 327-340. 
